\title{
Assessing the overall medication use by elderly people in a Brazilian hospital using the start/stopp criteria version 2
}

\author{
Thayná Ferreira Furtado Pereira ${ }^{1}$, Alessandra de Sá Soares ${ }^{2}$, Daisson José Trevisol ${ }^{3}$, \\ Fabiana Schuelter-Trevisol ${ }^{3}$
}

\begin{abstract}
${ }^{1}$ Universidade do Sul de Santa Catarina, Curso de Graduação em Medicina da Universidade do Sul de Santa Catarina (Unisul), Tubarão, Santa Catarina, Brasil, ${ }^{2}$ Universidade do Sul de Santa Catarina, Curso de Graduação em Farmácia e Bioquímica da Universidade do Sul de Santa Catarina (Unisul), Tubarão, Santa Catarina, Brasil, ${ }^{3}$ Universidade do Sul de Santa Catarina, Programa de Pós-Graduação em Ciências da Saúde da Universidade do Sul de Santa Catarina (Unisul) e Centro de Pesquisas Clínicas do Hospital Nossa Senhora da Conceição, Tubarão, Santa Catarina, Brasil
\end{abstract}

\begin{abstract}
To estimate the frequency of the use of medicines listed in the Screening Tool to Alert Doctors to the Right Treatment (START) and Screening Tool of Older Person's Prescriptions (STOPP) criteria version 2 among the elderly. A cross-sectional study was conducted on elderly who were attended in medical clinic and cardiology sectors in a hospital in southern Brazil attended at a hospital from February through September 2016. A data-collection tool was used to obtain information on variables, such as demographic and clinical data, and medications used before and during the hospitalization period. The adequacy of the medicines taken was examined with regard to omission (START) or inappropriate use (STOPP). This study was approved by the Research Ethics Committee of the University of Southern Santa Catarina. A total of 307 subjects were included in the final sample. The mean age was 75.2 years $(\mathrm{SD}=8$; range $65-102)$. Of the total, $93.5 \%$ had had at least one potential prescribing omission (PPO) listed in the START criteria, whereas $95.4 \%$ used at least one medicine of the STOPP criteria. PPO was significantly associated with lower mean age ( 74.9 years, $\mathrm{SD}=7.9$ versus 79.0 years, $\mathrm{SD}=8.8$ ) among the elderly who did not have PPOs detected by the START criteria (p-value=0.03). Furthermore, PPO was associated with longer hospital stay ( 18 versus 9 days; $p$-value $=0.03$ ). This study revealed inadequate prescription affecting $99.3 \%$ of the participating patients. To the best of our knowledge, this was the first to use the START and STOPP criteria, version 2, in Brazil.
\end{abstract}

Keywords: Drug utilization. Potentially inappropriate Medication list. Aged. Polypharmacy.

\section{INTRODUCTION}

In recent years, there has been a significant increase in the elderly population in all countries, which is attributed to the epidemiological transition phenomenon (Kanasi, Ayilavarapu, Jones, 2000; WHO, 2015). Longevity brings with it chronic disease and increased health resource utilization. The most commonly used health intervention for the elderly population is the administration of medicines (Menesier, Menicier-Ossia, 2017). However, polypharmacy can lead to the irrational use of medicines (Sivagnanam, 2016). Pharmacological treatments should

*Correspondence: F. Schuelter-Trevisol. Universidade do Sul de Santa Catarina (Unisul). Avenida José Acácio Moreira, 787, Tubarão - Santa Catarina - Brasil - CEP 88704-900. Tel.: (+55 048) 3631-7062. E-mail: fastrevisol@gmail.com iD be used with special caution among the elderly people, given that their organism presents physiological changes, such as decreased muscle mass and body water, as well as changes in the absorption, distribution, metabolism, and excretion of the medicines. Therefore, pharmacotherapy should be differentiated for the elderly, with caution in the prescription of medicines (Sánches-Fidalgo et al., 2017; Caughey et al., 2017).

Given the potentially serious consequences resulting from inadequate prescriptions (Prybys, Gee, 2002), some tools have been created for the use of medicines in the elderly. These include the Screening Tool to Alert doctors to the Right Treatment (START) and Screening Tool of Older Persons' potential inappropriate Prescriptions (STOPP). Both were published in 2008 by a panel of 18 experts in geriatric pharmacology in Ireland and the United 
Kingdom (Barry el al., 2007; Gallagher, O’Mahony, 2008).

The START/STOPP criteria are based on the list of potentially prescribing omission (PPO) and potentially inappropriate medicines (PIM) in clinical practice, respectively Barry el al. (2007) and Gallagher and O'Mahony (2008). In 2015, the second version expanded to 114 criteria: 34 START criteria and 80 STOPP criteria (O'Mahony et al., 2015). The criteria take into account pharmacological interactions, contraindications, precautions, and therapeutic duplications. This European instrument aims to rationalize the use of medicines. It acts in the prevention of adverse reactions, reducing unnecessary hospital admissions and, as a consequence, decreases public health expenditures (O'Mahony et al., 2015; Hill-Taylor et al., 2013).

The use of inappropriate medicines among elderly causes important health impact and assistance to this population. The results could highlight the need to develop and implement national strategies to identify inadequate prescriptions by creating and validating a Brazilian list.

The aim of this study was to estimate the use prescription frequency of medicines listed in the START/ STOPP criteria version 2 for hospitalized elderly.

\section{METHOD}

A cross-sectional epidemiological study was conducted on the medicines used by elderly patients assisted by the Brazilian Unified Health System (SUS), who were hospitalized in the medical clinic and cardiology sectors in a general hospital, located in southern Brazil. The survey was performed from February through September 2016. The sample size estimation took into account the total number of elderly patients hospitalized in the year 2015 , resulting in an estimate of 1,024 hospitalizations. A 50\% unknown prevalence for the outcome and a 5\% margin of error were used, resulting in a minimum sample of 280 patients for a $95 \%$ confidence level.

The sample was selected from the total number of hospitalizations during the survey period, and performed weekly by using the Tasy ${ }^{\circledR}$ system, a software designed for the electronic recording of medical records. Sampling by demand was engaged to recruit hospitalized elderly people 65 years of age and over. Those unable to answer the questions because of their clinical condition or discharged before the interview were excluded from the study.

At the first of second day of hospitalization, the patients were invited to participate in the study. Those who agreed, were asked to sign a free consent form. Then, they were interviewed by a trained researcher to collect sociodemographic and clinical data, and respond to questions about the medications they had taken at home the week before. At the end of the hospitalization period, the electronic medical records were reviewed to examine the prescriptions during the hospital stay and accounts of whether they were discharged or died.

The data-collection tool was designed to obtain information on targeted variables, such as gender, age, education, comorbidities, reasons for hospitalization, vaccination against Influenza and pneumococcus, continuous-use medicines and medication administered during hospitalization, listed by their generic names, classified according to the Anatomical Therapeutic Chemical (ATC) (WHO, 2016). The continuous-use medicines taken at home was checked through the presentation of packages, prescriptions, or reported by the patient or companion.

The START/STOPP criteria version 2 were employed to assess medication use in the target population (O'Mahony et al., 2015). The START tool is based on 34 criteria that indicate the use of certain medicines classes, based on the diagnosis and clinical status of elderly persons. The STOPP tool is composed of 80 criteria of medicines that should not be prescribed or taken by the elderly, except in special situations, at the physician discretion.

Based on the variables obtained, the adequacy of the medicines taken at home and at the hospital was examined with regard to omission (START) or inappropriate use (STOPP). In this study, 22 of the 34 START criteria and 16 of the 80 STOPP criteria were used. The fact that not all criteria were employed in the analysis was due to the lack of information related to complementary tests, medicines, and diseases that were not reported by the patients.

The collected data were entered into the Microsoft Excel 2010 Sheet and analyzed using the SPSS statistical package, version 20.0 (IBM, Armonk, New York, USA). To verify the association between START/STOPP criteria and variables of interest (such as mean age, time of hospitalization, number of medicines used and comorbidities) the chi-square test was applied, and the Student's t-test was used for mean comparison. When the Kolmogorov-Smirnov test revealed non-normal distribution, the Wilcoxon Mann-Whitney U test was applied. The statistical significance level was set at 0.05 .

This study was approved by the Research Ethics Committee of the University of Southern Santa Catarina (Opinion $\mathrm{N}^{\circ}$ 1.207.706).

\section{RESULTS}

A total of 337 elderly persons were recruited for this 
study. Of these, $30(8.9 \%)$ patients were excluded due to hospital discharge before the interview, which resulted in a final sample of 307 subjects. Mean age was 75.2 ( $\mathrm{SD}=8$ years), ranging from 65 to 102 years. The total number of medications taken at home by the participants was 1,832 , mean 6 medicines per patient. During the hospital stay, 4,617 medicines were prescribed, mean 15 medicines per elderly patient.

Table I presents the characteristics of the surveyed elderly.

Mean length of hospital stay was 17.5 days $(\mathrm{SD}=19.9)$, with a median of 11 days. Cardiovascular and respiratory disorders were the major reasons for admission

TABLE I - Characteristics of the surveyed elderly hospitalized, from February through September 2016. Tubarão (SC), Brazil. $(\mathrm{n}=307)$

\begin{tabular}{lc}
\hline Characteristics & $\mathbf{n}(\mathbf{\%})$ \\
\hline Age (years) & $98(31.9)$ \\
$65-69$ & $62(20.2)$ \\
$70-74$ & $61(19.9)$ \\
$75-79$ & $86(28.0)$ \\
$\geq 80$ & \\
Gender & $134(43.6)$ \\
Male & $173(56.4)$ \\
Female & \\
Education & $34(11.1)$ \\
Illiterate & $253(82.4)$ \\
Primary school & $18(5.9)$ \\
Secondary school & $2(0.6)$ \\
College degree & \\
Time of hospitalization (days) & $22(7.2)$ \\
1-3 & $65(21.2)$ \\
4-7 & $109(35.5)$ \\
$8-15$ & $111(36.1)$ \\
$>15$ & $104(6.9)$ \\
Comorbities* (n=1,509) & $488(32.3)$ \\
Respiratory disease & $200(13.3)$ \\
Cardiovascular disease & $254(16.8)$ \\
Gastrointestinal disorders & $140(9.3)$ \\
Metabolic disease & $170(11.3)$ \\
Nervous system disease & $153(10.1)$ \\
Muscoloskeletal disease & \\
Other & \\
\hline
\end{tabular}

*some patients had more than one comorbities. to the hospital. Of the participants, 64 (20.8\%) died during the hospital stay.

Table II shows the distribution of the medicines taken at home $(\mathrm{n}=1,832)$ and hospital $(\mathrm{n}=4,617)$, according to the ATC classification.

The most common classes of medicines taken by the elderly before and after hospital admission were those of groups $\mathrm{C}, \mathrm{A}$, and $\mathrm{N}$, related to the cardiovascular, digestive-metabolic and nervous systems, accounting for $67.0 \%$ of all administered medications.

Of the 307 surveyed elderly, $99.3 \%$ were affected by potentially inappropriate prescribing, of whom $93.5 \%$ had at least one medicine omission in the START criterion, and $95.4 \%$ had used at least one STOPP medicine. With regard to START, $85.0 \%$ of the medicines were omitted in the home treatment and $90.6 \%$ during the hospital stay. With regard to STOPP medicines, $54.4 \%$ were taken at home, and $94.1 \%$ were administered in the hospital. The mean number of potentially inappropriate medicines per patient was $1.6(\mathrm{SD}=0.7)$ of the medications used at home, ranging from 1 to 4 , and $2.8(\mathrm{SD}=1.5)$ of the medicines administered in the hospital, ranging from 1 to 8 .

Of the 344 different types of medicines taken by the surveyed elderly, 43 were potentially inappropriate medicines, and 27 were from the list of omitted medicines. The remaining 274 medicines were not classified by the START/STOPP criteria, either because they did not belong in this classification, or because of the lack of information that could allow a full evaluation.

Table III presents the five most commonly omitted medicines in the clinical practice at home $(\mathrm{n}=642)$ and at the hospital $(\mathrm{n}=582)$ by the surveyed elderly, according to the START criterion.

Regarding the PPOs according to the START criterion, patients with a lower mean age $(74.9, \mathrm{SD}=7.9$ years) had a larger number of medicines omissions as compared to patients with a higher mean age (79.0, $\mathrm{SD}=$ 8.8 years $),(p=0.03)$. Furthermore, prescribing omissions were associated with a longer hospital stay, (18 days versus 9 days $),(p=0.03)$.

The START criteria also showed a statistically significant association with coronary, cerebral or peripheral vascular disease $(\mathrm{p}=0.039)$, depression $(\mathrm{p}=$ 0.004 ), diabetes mellitus ( $<<0.001)$, chronic obstructive pulmonary disease $(\mathrm{p}=0,047)$, and systemic arterial hypertension $(\mathrm{p}<0.001)$.

Regarding immunoprophylaxis, $26.1 \%$ of the patients reported that they had not taken the vaccine against Influenza, and $89.9 \%$ against pneumococcus. These vaccines are indicated for elderly patients and are listed in the START criterion. 
TABLE II - Distribution of the medicines taken by the surveyed elderly hospitalized, from February through September 2016, according to the ATC classification. Tubarão (SC), Brazil

\begin{tabular}{llcc}
\hline \multirow{2}{*}{ Code } & Contents & \multicolumn{2}{c}{$\begin{array}{c}\text { Prescription frequency } \\
\text { n (\%) }\end{array}$} \\
\hline A & Alimentary tract and metabolism & Home & Hospital \\
\cline { 3 - 4 } B & Blood and blood forming organs & $355(19.3)$ & $950(20.6)$ \\
C & Cardiovascular system & $235(12.8)$ & $530(11.5)$ \\
D & Dermatologicals & $767(41.9)$ & $1,120(24.2)$ \\
G & Genitourinary system and sex hormones & $7(0.4)$ & $193(4.2)$ \\
H & Systemic hormonal preparations, excluding sex hormones and insulins & $3(0.3)$ & $18(0.4)$ \\
J & Antiinfectives for systemic use & $3(0.2)$ & $102(2.2)$ \\
L & Antineoplastic and immunomodulating agents & $22(1.2)$ & $377(8.2)$ \\
M & Musculoskeletal system & $8(0.4)$ & $10(0.2)$ \\
N & Nervous system & $28(1.5)$ & $46(1.0)$ \\
P & Antiparasitic products, insecticides and repellents & $312(17.0)$ & $814(17.6)$ \\
R & Respiratory system & $0(0.0)$ & $9(0.2)$ \\
S & Sensory organs & $84(4.6)$ & $425(9.2)$ \\
V & Various & $3(0.2)$ & $20(0.4)$ \\
Total & & $3(0.2)$ & $3(0.1)$ \\
\hline
\end{tabular}

TABLE III - Frequency of the five most commonly omitted medicines either at home or at the hospital by the surveyed elderly from February through September 2016, of those considered potentially appropriate according to the START criterion. Tubarão (SC), Brazil

\begin{tabular}{lc}
\hline Major potentially appropriate medicines omitted from the clinical practice, according to the START criteria & n (\%) \\
\hline At home (n=642) & $237(36.9)$ \\
\hline Antihypertensive therapy - if there was a history of systemic arterial hypertension & $112(17.4)$ \\
$\beta 2$ agonist or inhaled corticosteroid - if there was a chronic obstructive pulmonary disease or asthma & $63(9.8)$ \\
Statins - if there was a history of coronary, cerebral or peripheral vascular disease & $46(7.2)$ \\
Tricyclic antidepressants - if there was a history of depression & $34(5.3)$ \\
Bisphosphonates, vitamin D or calcium - if there was osteoporosis & $244(41.9)$ \\
\hline At the hospital (n=582) & $95(16.3)$ \\
\hline Antihypertensive therapy - if there was a history of systemic arterial hypertension & $49(8.4)$ \\
Laxatives - in patients receiving opioids regularly & $43(7.4)$ \\
Statins - if there was a history of coronary, cerebral or peripheral vascular disease & $33(5.7)$ \\
Tricyclic antidepressant - if there was a history of depression & \\
Bisphosphonates, vitamin D or calcium - if there was osteoporosis &
\end{tabular}

Table IV presents the frequency of the five potentially inappropriate medicines most commonly taken at home $(\mathrm{n}=318)$ and at the hospital $(\mathrm{n}=799)$, according to the STOPP criterion.

Omeprazole, clonazepam, and furosemide were the most commonly used medicines at home, accounting for $12.3 \%$ of those considered potentially inappropriate. At the hospital, the most commonly used medicines were omeprazole, furosemide, and tramadol, accounting for $25.0 \%$ of those considered potentially inappropriate. 
TABLE IV - Frequency of the five most commonly prescribed medications taken at home and at the hospital by the surveyed elderly, from February through September 2016, considered potentially inappropriate, according to the STOPP criterion, 2016. Tubarão (SC), Brazil

\begin{tabular}{lc}
\hline Major potentially inappropriate drugs prescribed according to STOPP criteria & n (\%) \\
\hline At home ( $\mathrm{n}=318)$ & $58(18.2)$ \\
\hline Omeprazole - If there was no history of gastroesophageal reflux disease, peptic ulcer, or gastritis & $43(13.5)$ \\
Clonazepam - Benzodiazepines can impair balance & $37(11.6)$ \\
Furosemide - If it was first line for systemic arterial hypertension & $32(10.1)$ \\
Spironolactone - Associated with angiotensin-converting enzyme inhibitor, angiotensin receptor blocker, or & $22(6.9)$ \\
amiloride & $129(16.1)$ \\
Glibenclamide - May cause hypoglycemia if taken for a long time & $81(10.1)$ \\
\hline At the hospital (n=799) & $70(8.8)$ \\
\hline Omeprazole - If there was no history of gastroesophageal reflux disease, peptic ulcer, or gastritis & $62(7.8)$ \\
Furosemide - If it was first line for systemic arterial hypertension & $50(6.3)$ \\
Tramal - Opioids without laxatives can cause chronic constipation & \\
Clonazepam - Benzodiazepines can impair balance & \\
Spironolactone - Associated with angiotensin-converting enzyme inhibitor, angiotensin receptor blocker, or & 5 \\
amiloride &
\end{tabular}

\section{DISCUSSION}

Medicines are considered potentially inappropriate for the elderly when there is no evidence-based clinical indication, and when they increase the risk of adverse effects or are not cost-effective (O’Mahony, Gallagher, 2008). Most study participants had at least one inadequate medicine prescription detected by the START/STOPP criteria. These findings are in line with other published works (Mori et al., 2017; Gallagher et al., 2011).

According to Pérez and colleagues, the definition of polypharmacy is controversial because it can mean the use of one medicine to correct the adverse effect of another, or taking five or more medicines simultaneously (Peréz et al., 2016). This study identified that the surveyed elderly took a large number of medicines (more than 5 per person), either before or after hospital admission, which confirmed the findings of other published works (Gutiérrez-Valencia et al., 2017; Ubeda et al., 2012).

With regard to the START criteria, antihypertensive medicines were the most commonly omitted prescribed PIMs, both at home and at the hospital, which means that antihypertensive therapy was not applied correctly, leading to therapeutic omission. In contrast to these results, other studies revealed that the most frequent therapeutic omissions occurred with statins, generally in patients with a history of coronary, cerebral or peripheral vascular disease (Anthierens et al., 2010; Olaniyan et al., 2015). Despite the different findings, in both cases, there was an underutilization of medicines, which may worsen the symptoms, disease control, and health status of the elderly (Anthierens et al., 2010). Furthermore, it may result in the need for additional prescription of larger doses or more potent therapies, which, in turn, may increase the risk of adverse reactions, as well as other negative outcomes, such as an increased number of visits to emergency services, hospitalizations, and deaths (Koper et al., 2013).

In this study, medicines omissions were associated with a lower mean age. Elderly patients with a lower age range may be more independent than those with a higher age range, which would explain the occurrence of medicine omission. In more advanced age groups, patients tend to need more medical care, which may explain a reduction in medicine omission. However, the risk of taking PIMs increases in the elderly (Marroquín et al., 2012). This scenario may result in an iatrogenic cascade, which occurs when the adverse effect of a medicine is misinterpreted as a new condition requiring repeated prescription, exposing the patient to possible additional detrimental effects related to pharmacological treatment (Garcia-Gollarte et al., 2012). These medication-related problems affect especially older people.

Patients who had a larger number of medicine omissions detected by the START criteria remained hospitalized twice as long as their counterparts. During hospitalization, patients are often treated by multiple physicians. As a consequence, their medicines prescriptions are vulnerable to iatrogenic effects, given 
that treatment is complex and involves a sequence of steps, such as prescription, communication, dispensing, administration, and clinical follow-up, which require more attention, especially in elderly patients (Saint-Germain et al., 2016).

With regard to the PPOs detected by the START criteria, there was a statistically significant association with coronary, cerebral, and peripheral vascular disease. It is well known that the leading causes of hospital admissions and deaths among the elderly are cardiovascular diseases, as previously mentioned. This study revealed that this comorbidity was neglected, given that the clinical situation required the prescription of medications, which was omitted. Furthermore, diabetes mellitus, depression, chronic obstructive pulmonary disease, and systemic arterial hypertension were also associated with prescribing omission. Such diseases, when not treated properly, may cause unfavorable fatal and nonfatal outcomes (Navar, Pencina, Peterson, 2016).

Influenza and pneumonia are the major causes of morbidity and mortality in patients with chronic obstructive pulmonary disease. Influenza vaccination reduces acute exacerbations, and the pneumococcal vaccine reduces the incidence of pneumonia (Di Giorgio, Provenzani, Polidori, 2016). Most of the participants in this study did not receive the anti-pneumococcal vaccine $(89.9 \%)$ and stated that they were not aware of this type of immunization. Moreover, $26.1 \%$ did not get a vaccination against Influenza. Both vaccines are recommended by the START criterion, and they should not be omitted. These data indicate the need for improvements in immunization campaigns, especially targeted at the elderly, as a strategy designed to increase prophylaxis adherence.

It should be noted that the use of medications was higher during hospitalization than at home. This fact can be explained by the fact that patients are admitted to the hospital in acute situations or when there are morbid complications derived from the aging process. Management of each case culminates in the prescription of more drugs to treat and control these clinical situations, in addition to continuous-use medications that must continue during the hospitalization period. These circumstances may increase the chances of using potentially inappropriate drugs or omission of others, in the absence of a medication reconciliation process. In fact, in the present study, the omission or inappropriate use of medications occurred more frequently in the hospital setting than at home.

With regard to the STOPP criterion, a comparison between medications taken at home and those taken at the hospital revealed that the prescription of PIMs increased during hospitalization. This distortion may be associated with the lack of regular review of the therapeutic regimen by health professionals. Strict compliance with clinical protocols should be the standard, otherwise the prescription of numerous medications may occur (Filkelstein et al., 2016b). In this context, it is worth mentioning that some studies have demonstrated that polypharmacy may increase the risk of PIM use (Sánchez-Fidalgo et al., 2017; Filkelstein et al., 2016a; Nascimento et al., 2014). A study conducted by Melo and colleagues (Melo, Storpirtis, Ribeiro, 2016) found that a medication therapy review by a clinical pharmacist resulted in a reduction in polypharmacy and PIMs after the hospitalization period.

With regard to the STOPP criteria, there was no statistically significant association between the use of PIM and the other variables collected. Because most patients took at least one PIM medication, and because the sample was homogeneous in relation to the outcome, the statistical analysis was compromised and could not show differences between the groups.

The most common PIMs taken at home or at the hospital, as detected by the STOPP criteria, belonged to the class of proton pump inhibitors, especially omeprazole. According to the STOPP criteria, this class of medicines should not be prescribed for patients who do not have a history of gastroesophageal reflux disease, peptic ulcer, or gastritis. These findings are consistent with those found in other studies (Cahir et al., 2010).

The findings from this study strengthen the need to create a national criterion on medication to be used by the elderly population, which would include potentially inappropriate medicines and those that should be prescribed specifically for this age group. This criterion would depict the Brazilian reality based on the list of essential medicines, whilst it will also provide guidance on rational prescription to healthcare professionals. The presence of clinical pharmacists both at the outpatient clinic and in the hospital setting is vital to help prescribers on the rational use of drugs, especially for the elderly population. A search software system and a list of potentially inappropriate medications are useful tools for prescribers and dispensers. It should be emphasized that the criteria applied will not prohibit the use of medicines, given that prescriptions will always depend on the situation and clinical decision, in addition to the individual characteristics of each patient (LozanoMontoya et al., 2015). This study neither evaluated the individual clinical history nor discussed the therapeutic choice with the attending physician. In addition, the results cannot be generalized since the study was conducted in a single center. 
It should be noted that not all START and STOPP criteria could be used in this study, because of the lack of clinical data and complementary tests, which was a limitation to the study. It should also be noted that there was a difference between the medications used at home, which were prescribed at an outpatient clinic, and those used in a hospital setting, with more complex clinical situations and less time available by the medical team to assess the safety of prescribed drugs, because of urgency in the acute situations.

In closing, this study was based on a representative sample and was the first to use the START and STOPP criteria, version 2, in Brazil to analyze medicines prescriptions before and after hospital admission. Further longitudinal studies should be conducted to investigate the risk factors associated with PIM and PPO and their consequences in order to provide a rational use of medicines among the elderly people.

\section{CONCLUSION}

The results from this study showed that potentially inappropriate prescribing affected $99.3 \%$ of the surveyed patients. Of the total prescriptions, $95.4 \%$ were related to inappropriate medicines, which could be suspended or modified according to the STOPP criterion, and 93.5\% were prescribing omissions of appropriate medicines, as detected by the START criterion. This study highlights the need to develop and implement national strategies to identify inadequate prescriptions by creating and validating a Brazilian list of medicines for the elderly people. Such a list should be widely disseminated to all health facilities to guide decisions about rational prescription and therapeutic use of medicines for the elderly population.

\section{REFERENCES}

Anthierens S, Tansens A, Petrovic M, Christiaens T. Qualitative insights into general practitioners' views on polypharmacy. BMC Fam Pract. 2010;11:65.

Barry PJ, Gallagher P, Ryan C, O'mahony D. START (Screening Tool to Alert Doctors tTo The Right Treatment) - an evidencebased screening tool to detect prescribing omissions in elderly patients. Age Ageing. 2007;36(6):632-8.

Cahir C, Fahey T, Teeling M, Teljeur C, Feely J, Bennett K. Potentially inappropriate prescribing and cost outcomes for older people: a national population study. Br J Clin Pharmacol. 2010;69(5):543-52.
Caughey GE, Tait K, Vitry AI, Shakib S. Influence of medication risks and benefits on treatment preferences in older patients with multimorbidity. Patient Prefer Adherence. 2017;11:131-40.

Di Giorgio C, Provenzani A, Polidori P. Potentially inappropriate drug prescribing in elderly hospitalized patients: an analysis and comparison of explicit criteria. Int J Clin Pharm. 2016;38(2):4628 .

Finkelstein J, Friedman C, Hripcsak G, Cabrera M. Pharmacogenetic polymorphism as an independent risk factor for frequent hospitalizations in older adults with polypharmacy: a pilot study. Pharmgenomics Pers Med. 2016a;9:107-16.

Finkelstein J, Friedman C, Hripcsak G, Cabrera M. Potential utility of precision medicine for older adults with polypharmacy: a case series study, Pharmgenomics Pers Med. 2016b;9:31-45.

Gallagher P, Lang PO, Cherubini A, Topinková E, Cruz-Jentoft A, Errasquín BM, et al. Prevalence of potentially inappropriate prescribing in an acutely ill population of older patients admitted to six European hospitals. J Clin Pharm Ther. 2011;67(11):117588 .

Gallagher P, O'Mahony D. STOPP (Screening Tool of Older Persons' potentially inappropriate Prescriptions): application to acutely ill elderly patients and comparison with Beers' criteria. Age Ageing. 2008;37(6):673-9.

García-Gollarte F, Baleriola-Júlvez J, Ferrero-López I, CruzJentoft AJ. Inappropriate drug prescription at nursing home admission. JAMA. 2012;13(1):83.e9-15.

Gutiérrez-Valencia M, Izquierdo M, Malafarina V, AlonsoRenedo J, González-Glaría B, Larrayoz-Sola B, MonforteGasque MP, Latasa-Zamalloa P, Martínez-Velilla N. Impact of hospitalization in an acute geriatric unit on polypharmacy and potentially inappropriate prescriptions: A retrospective study. Geriatr Gerontol Int. 2017;17(12):2354-2360.

Hill-Taylor B, Sketris I, Hayden J, Byrne S, O’Sullivan D, Christie R. Application of the STOPP/START criteria: a systematic review of the prevalence of potentially inappropriate prescribing in older adults, and evidence of clinical, humanistic and economic impact. J Clin Pharm Ther. 2013;38(5):360-72.

Kanasi E, Ayilavarapu S, Jones J. The aging population: demographics and the biology of aging. Periodontol 2000. 2016;72(1):13-8. 
Koper D, Kamenski G, Flamm M, Böhmdorfer B, Sönnichsen A. Frequency of medication errors in primary care patients with polypharmacy. Fam Pract. 2013;30(3):313-9.

Lozano-Montoya I, Vélez-Diaz-Pallarés M, Delgado-Silveira E, Montero-Errasquin B, Cruz Jentoft AJ. Potentially inappropriate prescribing detected by STOPP-START criteria: are they really inappropriate? Age Ageing. 2015;44(5):861-6.

Marroquín EC, Iglesia NM, Cobos LP. Adecuación de la prescripción farmacêutica en personas de 65 años o más en centros de salud docentes de Cáceres. Rev Esp Salud Pública. 2012;86(4):419-34.

Melo DO, Storpirtis S, Ribeiro E. Does hospital admission provide an opportunity for improving pharmacotherapy among elderly inpatients? Braz J Pharm Sci. 2016;52(3):391-401.

Menecier P, Menecier-Ossia L. Medicines and elderly patients: abuse, dependency or attraction. Soins Gerontol. 2017;22(123):16-20.

Mori AL, Carvalho RC, Aguiar PM, de Lima MG, Rossi MD, Carrillo JF, Dórea EL, Storpirtis S. Potentially inappropriate prescribing and associated factors in elderly patients at hospital discharge in Brazil: a cross-sectional study. Int J Clin Pharm. 2017;39(2):386-93.

Nascimento MMG, Ribeiro AQ, Pereira ML, Soares AC, LoyolaFilho AI, Dias-Júnior CAC. Identification of inappropriate prescribing in a Brazilian nursing home using STOPP/START screening tools and the Beers' Criteria. Braz J Pharm Sci. 2014;50(4):911-8.

Navar AM, Pencina MJ, Peterson ED. Assessing cardiovascular risk to guide hypertension diagnosis and treatment. JAMA Cardiol. 2016;1(8):864-71.

O'Mahony D, O'Sullivan D, Byrne S, O'Connor MN, Ryan C, Gallagher P. STOPP/START criteria for potentially inappropriate prescribing in older people: version 2. AgeAgeing. 2015;44(2):2013-8.
Olaniyan JO, Ghaleb M, Dhillon S, Robinson P. Safety of medication use in primary care. Int J Pharm Pract. 2015;23(1):320.

O'Mahony D, Gallagher PF. Inappropriate prescribing in the older population: need for new criteria. Age Ageing. 2008;37(2):138-41.

Pérez MDC, Alcalá MVC, Matas RQ, Martín SM, Pascual CM, Puime AO. Use of primary care services, care specialized and drug use by population 65 years and more in Madrid, Spain. Rev Esp Salud Pública. 2016;26(90):e1-11.

Prybys KM, Gee A. Polypharmacy in the elderly: clinical challenges in emergency practice: part I: overview, etiology, and drug interactions. Emerg Med Rep. 2002;23(11):145-53.

Saint-Germain P, Ruelle M, Mary A, Sid Idris S, Hannat S, Pelloquin N, Jouanny P, Terrier-Lenglet A. The clinical impact of treatment discrepancies recorded for 200 patients in an acute geriatric unit. Rev Med Interne. 2016;37(10):667-73.

Sánchez-Fidalgo S, Guzmán-Ramos MI, Galván-Banqueri M, Bernabeu-Wittel M, Santos-Ramos B. Prevalence of drug interactions in elderly patients with multimorbidity in primary care. Int J Clin Pharm. 2017;39(2):343-53.

Sivagnanam G. Deprescription: The prescription metabolism. J Pharmacol Pharmacother. 2016;7(3):133-7.

Ubeda A, Ferrándiz L, Maicas N, Gomez C, Bonet M, Peris JE. Potentially inappropriate prescribing in institutionalized older patients in Spain: the STOPP-START criteria compared with the Beers criteria. J Pharm Pract, 2012;10(2):83-91.

WHO collaborating centre for drug statistics methodology. [Acesso em: 23 Ago 2016]. Disponível em: http://www.whocc. no/atc_ddd_index/.

World Health Organization. World Report on Ageing and Health. 2015. [cited $2017 \mathrm{Mar}$. Available in: http://apps.who.int/iris/ bitstream/10665/186463/1/9789240694811_eng.pdf.

Received for publication on $25^{\text {th }}$ November 2017 Accepted for publication on $12^{\text {th }}$ June 2018 